\title{
Neonatal exposure to hyperoxia leads to persistent disturbances in pulmonary histone signatures associated with NOS3 and STAT3 in a mouse model
}

Cho-Ming Chao ${ }^{1,2}$, Rhea van den Bruck 3 , Samantha Lork³, Janica Merkle ${ }^{3}$, Laura Krampen³, Patrick P Weil ${ }^{3}$, Malik Aydin ${ }^{3}$, Saverio Bellusci', Andreas C. Jenke ${ }^{3,4^{*}+}$ (D) and Jan Postberg ${ }^{3+}$

\begin{abstract}
Background: Early pulmonary oxygen exposure is one of the most important factors implicated in the development of bronchopulmonary dysplasia (BPD).

Methods: Here, we analyzed short- and long-term effects of neonatal hyperoxia on NOS3 and STAT3 expression and corresponding epigenetic signatures using a hyperoxia-based mouse model of BPD.

Results: Early hyperoxia exposure led to a significant increase in NOS3 (median fold change $\times 2.37$, IQR 1.54-3.68) and STAT3 (median fold change $\times 2.83$, IQR 2.21-3.88) mRNA levels in pulmonary endothelial cells with corresponding changes in histone modification patterns such as H2aZac and H3K9ac hyperacetylation at the respective gene loci. No complete restoration in histone signatures at these loci was observed, and responsivity to later hyperoxia was altered in mouse lungs. In vitro, histone signatures in human aortic endothelial cells (HAEC) remained altered for several weeks after an initial long-term exposure to trichostatin A. This was associated with a substantial increase in baseline eNOS (median 27.2, IQR 22.3-35.6) and STAT3a (median 5.8, IQR 4.8-7.3) mRNA levels with a subsequent significant reduction in eNOS expression upon exposure to hypoxia.
\end{abstract}

Conclusions: Early hyperoxia induced permanent changes in histones signatures at the NOS3 and STAT3 gene locus might partly explain the altered vascular response patterns in children with BPD.

\section{Background}

Bronchopulmonary dysplasia (BPD) is a chronic lung disease of prematurely born infants and remains a leading cause of morbidity and mortality. Currently, there is no curative therapy available. Based on the severitybased definition of BPD (inclusion of infants with mild $\mathrm{BPD}), 68 \%$ of premature infants born with a gestational age $(\mathrm{GA}) \leq 28$ weeks develop BPD [1-3]. The risk of developing $\mathrm{BPD}$ correlates inversely with the gestational

\footnotetext{
* Correspondence: Andreas.jenke@uni-wh.de

${ }^{\dagger}$ Equal contributors

${ }^{3}$ Department of Pediatrics, HELIOS Medical Center Wuppertal, Center for Clinical \& Translational Research (CCTR), Center for Biomedical Education \& Research (ZBAF), Witten/Herdecke University, Wuppertal, Germany ${ }^{4}$ EKO Children's Hospital, Oberhausen, Witten/Herdecke University, Alfred-Herrhausen Str. 40, Witten, Germany

Full list of author information is available at the end of the article
}

age (GA) and birth weight (BW) [4]. Since premature infants are born with a lung which is in the canalicular or saccular stages of development, the lung structure is therefore not adequate to provide sufficient ventilation and gas exchange. Thus, mechanical ventilation and high oxygen concentration are often necessary at birth. Barotrauma induced by mechanical ventilation as well as oxygen toxicity and inflammation are major contributing factors responsible for the pulmonary damages in the immature lung. In addition, some studies have suggested a strong genetic component in BPD [5]. In fact, clinical studies suggest that decisions during the first minutes of life [6] or even events before the delivery might be crucial for the later development of BPD. In line with these clinical data are observations that hyperoxia in rat pups leads to increased endothelilal nitric oxide synthase (eNOS) 
levels, nitric oxide (NO) activity, hyperemia [7, 8], and possibly eNOS uncoupling eventually leading to BPD.

Importantly, BPD does not only affect neonatal mortality but also leads to long-term morbidity, e.g., increased susceptibility to upper respiratory infections during the first year of life [9] and pulmonary hypertension [10]. Again, neonatal oxygen seems to be an important pathological factor since it increases for example the sensitivity to influenza A virus infection by suppressing epithelial expression of Ear1 [11]. In fact, extremely and moderately preterm infants face a 3.6 times increased risk of being hospitalized due to respiratory infection in the first year of life [12].

Whereas it seems clear from twin studies that genetic susceptibility plays an important role for disease development [13], the exact mechanism remains unclear but epigenetic mechanisms seem to be part of it since several recent publications have reported abnormalities of histone acetylase activity and the chromatin remodeling pathway in BPD patients $[14,15]$. Thus, persistent changes in epigenetic signatures might be also at least partly responsible for the later development of pulmonary hypertension and the increased susceptibility to respiratory tract infections. Since we have recently shown that epigenetic signatures at NOS3 encoding eNOS in human umbilical artery endothelial cells are substantially shaped by prenatal events such as placental insufficiency and that there exists an interdependency between NOS3 and the gene activity of signal transducer and activator of transcription 3 alpha (STAT3 $\alpha$ ) as well as Stat3transcription factor-binding in the NOS 3 promoter. Moreover, in human, a 27-nt non-coding RNA becomes co-expressed with NOS3 and post-transcriptionally regulates STAT3 expression [16]. We thus aimed to utilize the same gene loci to analyze the effect of early neonatal oxygen exposure on the pulmonary vascular endothelial epigenetic landscape and the associated consequences for oxygen exposure later in life.

Therefore, we analyzed NOS3 gene expression in response to early neonatal hyperoxia at first and then studied the respective changes in epigenetic signatures as consequences of this first early oxygen exposure on later events of hyperoxia and hypoxia later in life.

\section{Methods \\ Mice}

Wildtype mice (males and females, CD1 background) were crossed to generate time-pregnant females. All mice received food and water ad libitum.

\section{Hyperoxia injury (BPD mouse model)}

Newborn pups were exposed to either normoxia (NOX) or hyperoxia (HOX) within $24 \mathrm{~h}$ after birth (P0) (Fig. 1). In experimental group 1 , dams and pups were kept in NOX from P0 to P16. In experimental group 2, dams and pups were kept in NOX from P0 to P15. From P15 to $\mathrm{P} 16$, the dams and the pups were exposed to HOX $\left(85 \% \mathrm{O}_{2}\right)$. In experimental group 3 , newborn pups were subjected to $\mathrm{HOX}\left(85 \% \mathrm{O}_{2}\right)$ injury from $\mathrm{P} 0$ to $\mathrm{P} 8$ in a chamber (Proox Model 110, Biospherix). To minimize oxygen toxicity and bias, nursing dams were rotated every $24 \mathrm{~h}$ between NOX and HOX. Afterwards, nursing dams and pups were exposed to NOX $\left(21 \% \mathrm{O}_{2}\right)$ from P8 to P16. From P15 to P16, the dams and the pups were re-exposed to $\mathrm{HOX}\left(85 \% \mathrm{O}_{2}\right)$. In experimental groups 1 and 3, lungs were harvested at P8. In all groups, lungs were harvested at P16. All dams and pups received food and water ad libitum.

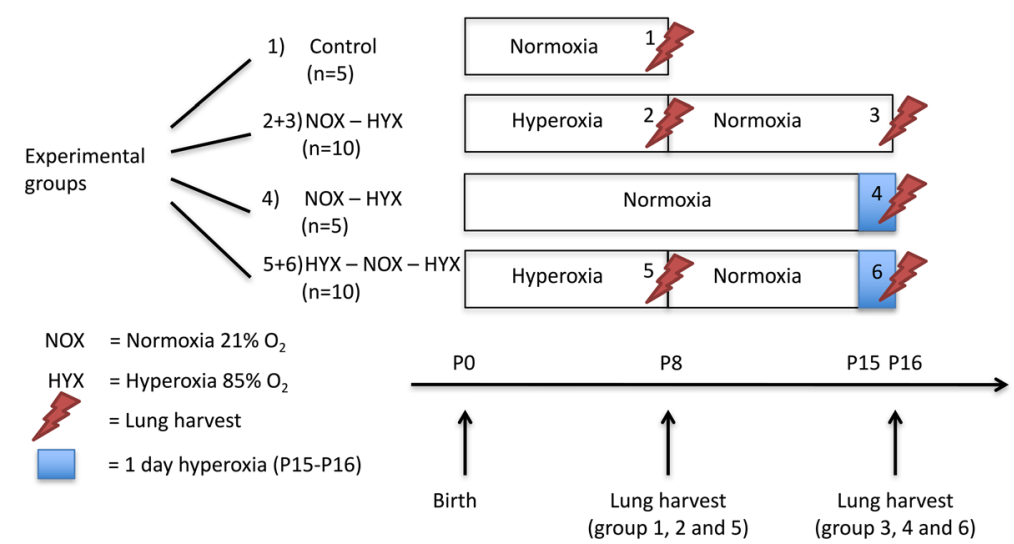

Fig. 1 Experimental setting. The experimental setting consists of six groups. Each group consists of five animals. The experiments were performed in two steps. The first comprised a control group at P8 in normoxia (group 1), a group after neonatal hyperoxia from P0 to P8 (group 2), and a group exposed to neonatal hyperoxia from P0 to P8 with eight additional days of normoxia until P16 (group 3). The second comprised a control group in normoxia at P16 (group 4), a group after neonatal hyperoxia from P0 to P8 (group 5), and a group exposed to neonatal hyperoxia (P0 to P8) followed by normoxia (P8 to P15), and a second exposure to hyperoxia for 1 day before analysis at P16 (group 6). P postnatal 
Lung perfusion, isolation, tissue processing, and histology Mice were euthanized by intraperitoneal injection of a solution made of ketamin, dormitor, heparin, and saline. After sternotomy, the lung was perfused transcardially (through the right ventricle) by using PBS $(1 \times)$, then isolated and incubated for $30 \mathrm{~min}$ at $4{ }^{\circ} \mathrm{C}$ in COLD medium, stored at $-20{ }^{\circ} \mathrm{C}$ overnight, and finally stored at $-80^{\circ} \mathrm{C}$ till further tissue processing.

For histology, the lung was flushed from the right ventricle to remove blood cells then perfused through the trachea with a pressure of $20 \mathrm{~cm} \mathrm{H}_{2} \mathrm{O}$ with $5 \mathrm{ml} 4 \%$ PFA. The trachea was tied off with a string, and the lung was removed and placed in $4 \%$ PFA for max. $24 \mathrm{~h}$ at $4^{\circ}$ C. Lungs were then progressively dehydrated $(30,50,70$, and $99 \%$ ethanol, $3 \mathrm{~h}$ each), incubated in xylole, then in paraffine overnight and finally embedded with a Leica embedding machine (EG 1150C). Paraffin blocks were kept cold, and $5 \mu \mathrm{m}$ sections were cutted. Hematoxylin and eosin staining was performed according to protocols previously published.

\section{Positive selection for vascular endothelial cells}

Positive selection for vascular endothelial cells was performed by magnetic separation with human CD146 Microbeads (Miltenyi ${ }^{\circ}$, Bergisch-Gladbach, Germany) following manufacturers' instructions with minor modifications as described previously [17].

\section{RNA isolation}

Total RNA was isolated using Trizol (Sigma-Aldrich) and isopropanol precipitation and further purification on columns. Next, RNA integrity was assayed using the Agilent Bioanalyzer 2000. Only samples with nonfragmented RNA were included.

\section{Gene expression analyses}

Gene expression analyses were performed using quantitative real-time PCR (qPCR) analyses on a Rotor-Gene 6000 (Qiagen). For PCR reactions, QuantiTect SYBR Green qPCR Master Mix (Qiagen) containing Hot Start Taq DNA polymerase and SYBR Green was used. Primers were used as described earlier [18]. The expression of gene of interest was normalized using three housekeeping genes (BACT, GAPDH, PECAM1). PCR conditions were as follows: $95{ }^{\circ} \mathrm{C}$ for $15 \mathrm{~min}, 40 \times\left[95{ }^{\circ} \mathrm{C}\right.$ for $15 \mathrm{~s}, 60{ }^{\circ} \mathrm{C}$ for $30 \mathrm{~s}$ ]. Melting of PCR product was done using a gradient from 55 to $95{ }^{\circ} \mathrm{C}$ rising in $0.5{ }^{\circ} \mathrm{C}$ increments.

\section{Antibodies}

Primary antibodies used in this study were (1) Rabbit anti-H2A.Zac (Diagenode pAb-173-050), (2) Rabbit antiH3K9ac (Active Motif pAb\#39137), and (3) Rabbit antiH3K4me3 (Diagenode pAbCSP-030-050).

\section{Chromatin purification and ChIP assays}

Chromation purification and ChIP assays were performed as described previously [15]. Quantitative PCR analyses were performed using a Rotorgene 6000 (Qiagen). The relative amounts of specifically immunoprecipitated DNA were estimated as "percent of input" and quantified using individual standard curves for each amplicon. Primer pairs were used as described earlier [18].

\section{Pyrosequencing}

DNA methylation signatures of promoter segments of NANOG, NFE2L2, and STAT3 were analyzed using a Qiagen Pyromark Q24 sequencer as previously described (Plos One Jenke et al.). Briefly, after standard sodium bisulfite conversion using the EZ DNA Methylation-Gold Kit (Zymo Research, USA) pyrosequencing methylation analysis was conducted using the PyroMark Q24 (Qiagen, Germany) according to the manufacturer's protocol. Therefore, we designed and made use of the following oligonucleotides: 1. for NFE2L2-primer F1 (5' - gga gtt aga ggg gat agt ggt t-3'), 5'-biotinylated primer ( $5^{\prime}$-acc cca cca at caa aac ttc ct-3'), and sequencing primer S1 (5'-agg gta aag gag gat g-3'); 2 . for STAT3-primer F1 (5'-ggt gta ggg tgg ggt tat $\left.\mathrm{t}-3^{\prime}\right)$, $5^{\prime}-$ biotinylated primer $\left(5^{\prime}\right.$-acc cta tat atc tcc tcc tat cct- $\left.3^{\prime}\right)$, and sequencing primer S1 (5'-ggg tgg ggt tat $\left.\mathrm{ttt} t \mathrm{t}-3^{\prime}\right)$; 3 . for NANOG (DNA methylation positive control)-primer F1 (5' -gta gga tag gaa tgg ggg ttg-3'), 5'-biotinylated primer $\left(5^{\prime}\right.$-acc tta aat tta ccc caa att cta c- $\left.3^{\prime}\right)$, and sequencing primer S1 (5'-aat ggg ggt tgg gga- $\left.3^{\prime}\right)$. No reliable NOS3 DNA methylation assays meeting our quality standards could be designed. The level of methylation was analyzed using PyroMark Q24 2.0.6 Software (Qiagen). Non-CpG cytosine residues and a standard fully methylated DNA (Zymo Research, USA) were used as controls to verify bisulfite conversion.

\section{Small RNA-seq and analyses pipeline}

Total RNA was purified as described above. For multiplexing, we made use of different multiplex sequencing barcodes for sequencing in a single lane as described [19]. Briefly, total RNA was separated by polyacrylamide gel electrophoresis. Gel fragments corresponding to 15 to 35 nt RNA molecules were cut, and RNA was eluted. These small RNAs were directly used for the construction of sequencing libraries in four steps: step 1: ligation of DNA oligos to the 3 '-end of the RNA; step 2: ligation of RNA or, respectively, chimeric RNA/DNA oligos to the 5'-end of RNAs; step 3: cDNA library synthesis by reverse transcriptase; and step 4: amplification of the cDNA library. Subsequently, after final quality checks by microcapillary electrophoresis and qPCR, the libraries were sequenced on an Illumina Hiseq 2000 platform 
(single end, $50 \mathrm{bp}$ ). This work has benefited from the facilities and expertise of the high-throughput sequencing core facility of IMAGIF Gif-sur-Yvette (Centre de Recherche de Gif-www.imagif.cnrs.fr). The initial data analysis pipeline was as follows: CASAVA-1.8.2 was used for demultiplexing, Fastqc 0.10 .1 for read quality assessment and Cutadapt-1.3 for adaptor trimming, resulting in an average sequence number for each developmental time point sample of $7.58 \mathrm{Mbp}$. File conversions, filtering, and sorting as well as mapping (Bowtie2) were done using "Galaxy" [20-22], a platform for data intensive biomedical research (https://usegalaxy.org/), and "Chimira" [23], respectively.

\section{Immunofluorescence staining for eNOS}

Paraffin sections were deparaffinized. Antigen retrieval was performed for $15 \mathrm{~min}$ in citrate buffer using a rice cooker, then slides were cooled down on ice for $20 \mathrm{~min}$. After washing slides three times in TBST (TBS buffer + $0.1 \%$ Tween 20) for $5 \mathrm{~min}$, slides were blocked with $3 \%$ bovine serum albumin (BSA) and $0.4 \%$ Triton X-100 [in Tris-buffered saline (TBS)] at room temperature (RT) for $1 \mathrm{~h}$ and then incubated with primary antibody against eNOS (ThermoFisher Scientific, PA1-037; 1:100) at $4{ }^{\circ} \mathrm{C}$ overnight. Afterwards, slides were again washed three times in TBST for $5 \mathrm{~min}$, incubated with secondary antibody (Goat Anti-Rabbit Alexa 555, ThermoFisher Scientific, A-21429; 1:500) in room temperature for $1 \mathrm{~h}$, and then washed three times in TBST before being mounted with Prolong Gold Anti-fade Reagent with DAPI (4,6-diamidino-2-phenylindole; ThermoFisher Scientific, P36931). Fluorescent images were acquired using Leica DM5500 B fluorescence microscope connected to Leica DFC360 FX camera.

\section{Statistical analysis}

Data were compared using Mann-Whitney $U$ test according to normality assumptions on univariate analysis followed by Bonferroni correction for multivariate analysis. Categorical variables were compared using the Fisher exact test. Statistical analyses were performed with GraphPad Prism 5.0.

\section{Cell culture}

Human arterial endothelial cells (HAEC) were bought from PromoCell (Heidelberg, Germany) and cultivated upon manufacturer's recommendations. For cDNA synthesis, we used 500 ng RNA per sample using the QuantiTect Reverse Transcription kit (Qiagen). DNA was isolated by phenol:chloroform:isoamylic alcohol extraction followed by precipitation with isopropanol. For in vitro experiments, HAEC (PromoCell) were cultivated upon manufacturer's recommendations. For HDAC inhibition, HAEC were treated with $1 \mu \mathrm{M}$ trichostatin $\mathrm{A}$
(TSA) for either $72 \mathrm{~h}$ or twice for $6 \mathrm{~h}$ with an 60 -h interval in between. Hypoxia experiments were performed using a hypoxia incubator chamber (STEMCELL Technologies, Grenoble, France) exposing cells to a ppO2 of 0.12 bar corresponding to an oxygen fraction of $12 \%$ for $24 \mathrm{~h}$. Control experiments were performed under normoxic conditions ( $\mathrm{ppO} 2=0.21$ bar).

\section{Results}

Neonatal hyperoxia from P0 to P8 leads to increased NOS3 expression-in both whole lung tissue and CD146positive cells

For our study, we used a well-established mouse model for bronchopulmonary dysplasia. Newborn pups were exposed to $85 \%$ oxygen from P0 to P8 then stayed in normoxia from P8 to P16. Lungs were analyzed at P8 and P16. In neonatal mouse lungs, exposure to hyperoxia (85\% oxygen) from P0 to P8 led to a significant increase in NOS3 (median fold change $\times 2.37$, IQR $1.54-3.68, p=0.003$ ), GPX1 (median fold change $\times 1.73$, IQR 1.28-2.08, $p=0.001$ ), and STAT3 $\alpha$ and $\beta$ (median fold change $\times 2.83$, IQR 2.21-3.88, $p=0.001$ ) mRNA levels at P8. Other genes, such as HIF1A for example, were not differentially expressed on the mRNA level (Fig. 2a). At P16, 8 days after hyperoxia, mRNA levels of GPX1 and STAT3 dropped to levels comparable to the normoxia group at P8. NOS3 mRNA levels also dropped but tended to be slightly above the level of the normoxia group at P8-even though this difference did not reach statistical significance except for primer pair matching to exon10/11 (Fig. 2a). In addition, we aimed to determine whether expression profiles upon oxygen exposure differ between whole lung tissue and pulmonary endothelial cells. Therefore, we isolated vascular endothelial cells from whole lung tissue using magnetic separation with human CD146 microbeads. Results obtained from CD146positive cell population are shown in Fig. 2b. For all analyzed genes of interest, we did not see any differences in expression profiles. After hyperoxia, histology showed simplification of alveoli with increased airspace and dilated alveoli (Fig. 3c, d) compared to the normoxia group (Fig. 3a, b) indicating hypoalveologenesis due to disturbed secondary septa formation. This could be associated with the vascular malformation seen in children with BPD [24, 25],

\section{Responsivity to later hyperoxia at P15 is altered in mouse lungs that have been exposed to hyperoxia in the neonatal period}

Exposure to hyperoxia later in life at P15 led to substantial differences in mRNA levels compared to mice previously exposed to hyperoxia during the neonatal period and those without. More specifically, there was less 

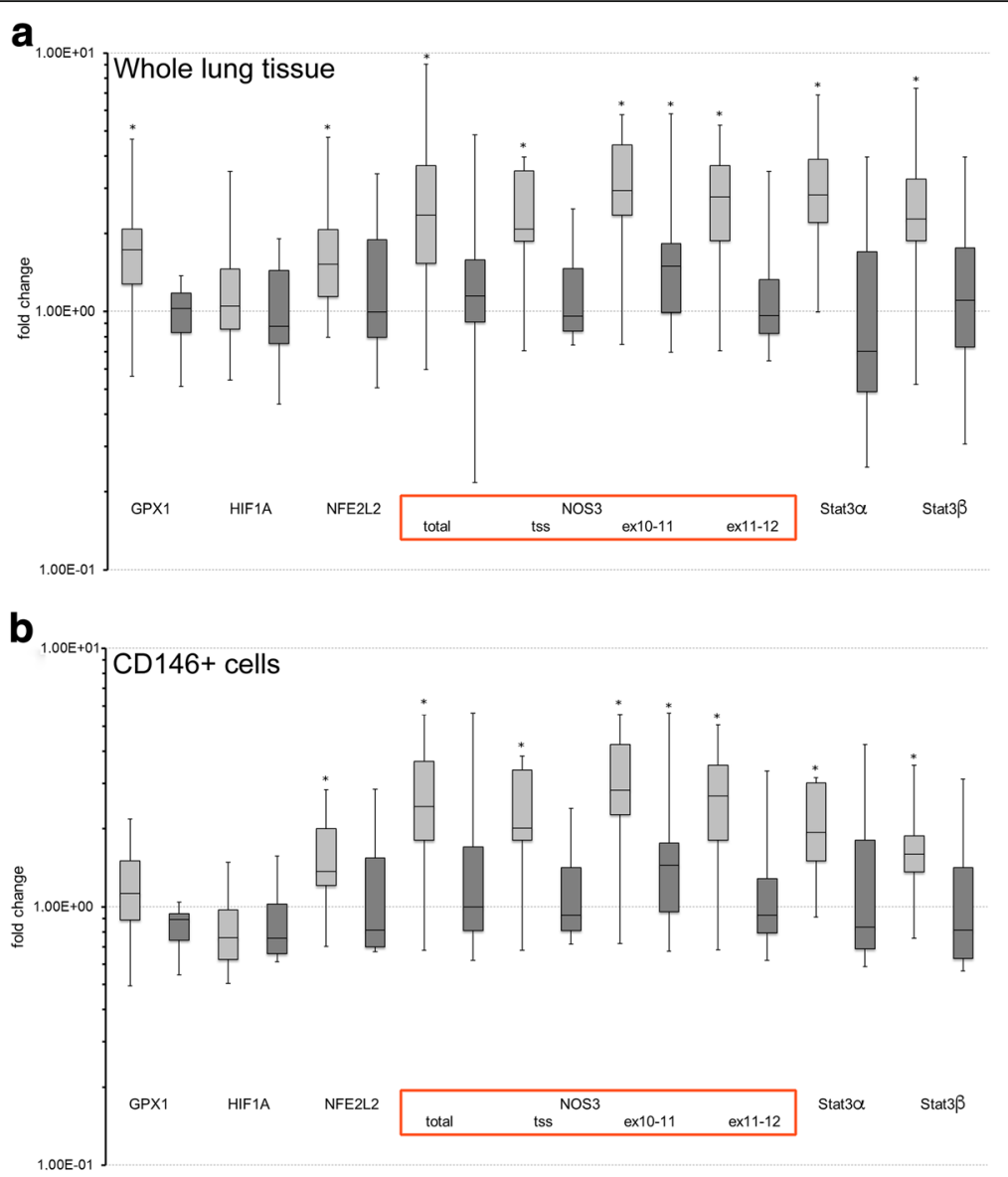

Fig. 2 Expression profile of whole lung tissue versus endothelial cells (CD 146+). The upper part shows expression patterns in whole lung tissue (a), the lower in CD146-positive endothelial cells (b). Light gray bars indicate mice immediately after hyperoxia (group 2), dark gray 8 days of normoxia after hyperoxia from P0 to P8 (group 3). Expression data is normalized to the control group 1. Every group contained five animals. Error bars indicated $95 \% \mathrm{Cl}$. Asterisks indicate significant difference to the control group $(p<0.05)$

increase in NOS3 mRNA levels (median fold change $x$ 1.90 , IQR $0.92-2.72$ ) in mice that were previously exposed to hyperoxia compared to mice exposed to normoxia (median fold change $\times 3.93$, IQR 2.50-8.51). Interestingly, the increase in STAT3 mRNA was similar in both groups-even though it tended to be higher in the hyperoxia (median 3.73, IQR 1.38-7.29) compared to the normoxia group (median 2.30, IQR 0.74-4.53) - when compared to the median fold change $(\times 2.83$, IQR $2.21-3.88)$ after the first exposure. With respect to the other genes investigated, such as NFE2L2 (Fig. 4c), GPX1, and HIF1A (data not shown), we found no significant differences between mice with and without previous hyperoxia exposure (Fig. 4). Since in human, a small 27 nt-RNA is apparently involved in the negative regulation of NOS3 mRNA via STAT3 mRNA targeting, we performed holistic analyses of microRNA (miR) profiles in purified lung epithelial cell obtained from experiments under normoxic (group 1), hyperoxic (groups 2/5), and after repetitive hyperoxic treatment (group 6). Notably, an ortholog of human 27 nt-RNA could not be identified in the mouse genome. Therefore, we aimed to identify differentially expressed murine candidate miRs, which potentially could target STAT3 mRNA in a way reminiscent of human 27 nt-RNA and human STAT3 mRNA. In total, we identified 301 different miRs from which 101 were non-marginally expressed and thus were considered for differential analyses. Figure $4 \mathrm{~d}$ shows the relative average enrichment of these $101 \mathrm{miRs}$ and below the group-specific quantitative profiles as a heat map. Interestingly, almost general drop of miR abundance was observed after hyperoxic treatment (groups $2 / 5$ ) when compared with normoxia (group 1), whereas the abundance of many miRs increases markedly, when a repetitive oxygen treatment was applied (group 6). Notably, we identified several miRs differentially overexpressed under these conditions, where strong evidence exists for STAT3 mRNA targeting in mice (Fig. 4e), i.e., mmu-let-7b-5p, mmu-miR-181a-5p, mmu-miR-93-5p, mmu-miR-17-5p, and mmu-miR125a-5p. 


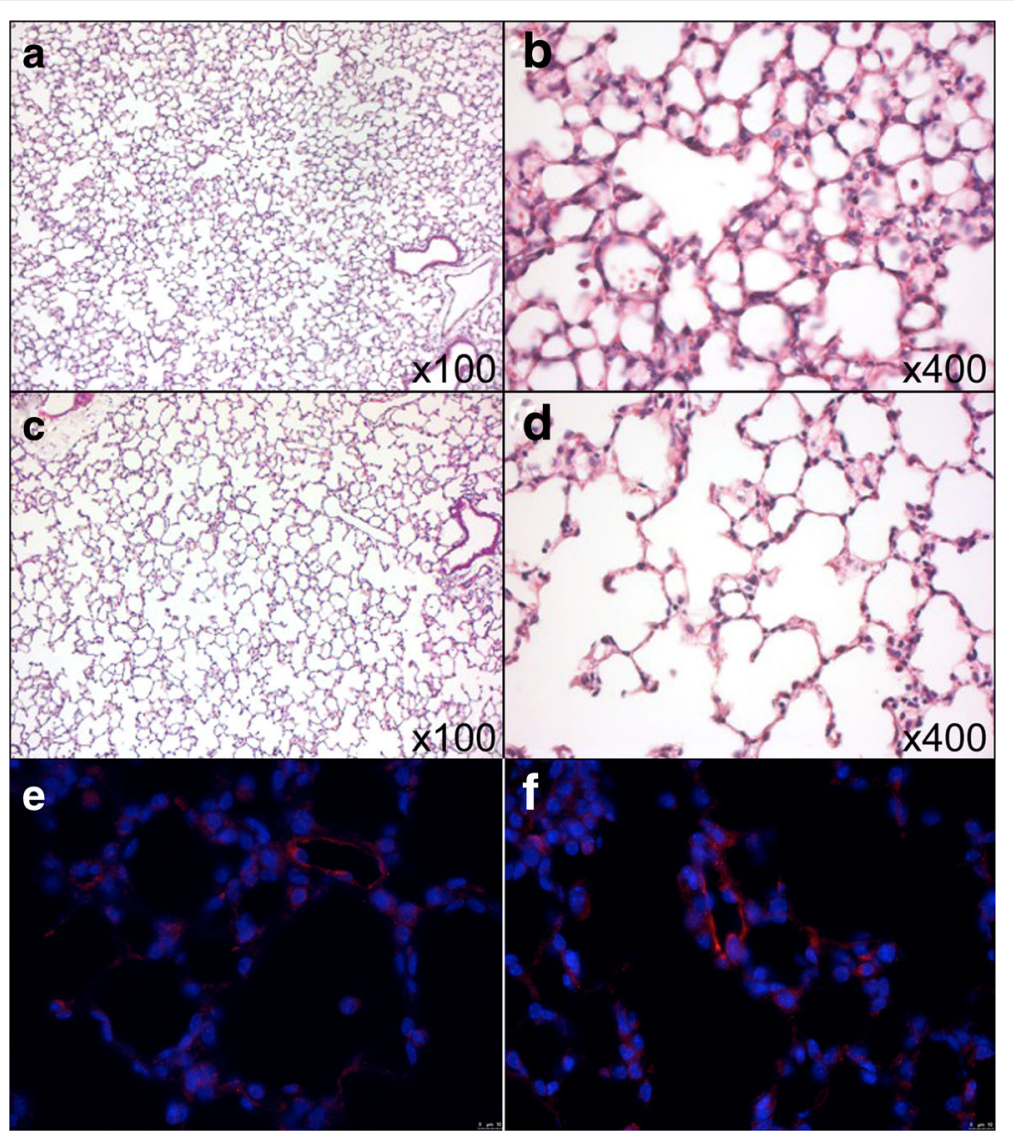

Fig. 3 Neonatal hyperoxia leads to hypoalveologenesis at P8. a, b The lungs of the normoxia group at P8 (group 1) show normal developed alveoli. c, d The lungs from the hyperoxia group (group 2) at P8 after exposure to 85\% oxygen from P0 to P8 reveal a significant hypoalveologenesis with dilated alveoli and increased airspace macroscopically due to disturbed secondary septa formation. H\&E. e, $\mathbf{f}$ Immunofluorescence staining on P8 comparing normoxia (e, group 1) versus hyperoxia (f, group 2) show higher expression after hyperoxia. $\mathbf{a}, \mathbf{c}$ $\times 100 ; \mathbf{b}-\mathbf{f} \times 400$

Modifications in histone signatures at the NOS3 and STAT3 gene locus in response to hyperoxia exposure are not restored

We then further analyzed in pulmonary vascular endothelial cells whether changes in histone and/or CpG signatures were associated with the observed expression patterns. Whereas we did not see any significant differences in CPG methylation (Table 1), histone modifications patterns at the NOS3 and STAT3 gene locus changed substantially. Upon initial exposure to hyperoxia, we noted an increase in $\mathrm{H} 2 \mathrm{aZac}$ at both loci and an additional decrease in H3K4me3 at the NOS3 locus. No significant alterations were observed for H3k9ac. Importantly, levels for H2aZac did not return to a baseline level but remained elevated and levels for H3K9ac increased significantly after 8 days of normoxia at both loci (Fig. 4a, b). Interestingly, upon second exposure to hyperoxia, we observed an even further increase in $\mathrm{H} 2 \mathrm{aZac}, \mathrm{H} 3 \mathrm{~K} 4 \mathrm{me}$, and $\mathrm{H} 3 \mathrm{~K} 9 \mathrm{ac}$ in mice with previous excessive oxygen exposure when compared to mice raised in a normoxic environment, which showed an acetylation/methylation pattern very similar to the initial pattern observed upon the primary excessive oxygen exposure (Fig. 4a, b). At the NFE2L2 locus, no changes were noted (Fig. 4c).

Long-lasting disturbances in histone modification at the NOS3 gene locus lead to disruption of the regulation of eNOS expression upon physiological stimuli later on To further analyze whether persistent histone modifications are primarily responsible for the altered eNOS response pattern of vascular endothelial cells upon physiological stimuli later on, we used human aortic endothelial cells (HAEC) which were exposed to trichostatin A (TSA). To mimic persistent environmental stimulation, we incubated HAEC with TSA for $72 \mathrm{~h}$ and compared these to unexposed HAEC and HAEC that were exposed twice for $6 \mathrm{~h}$ with an interval of $60 \mathrm{~h}$ in between to simulate a more physiological situation. Whereas persistent stimulation led to a change in 


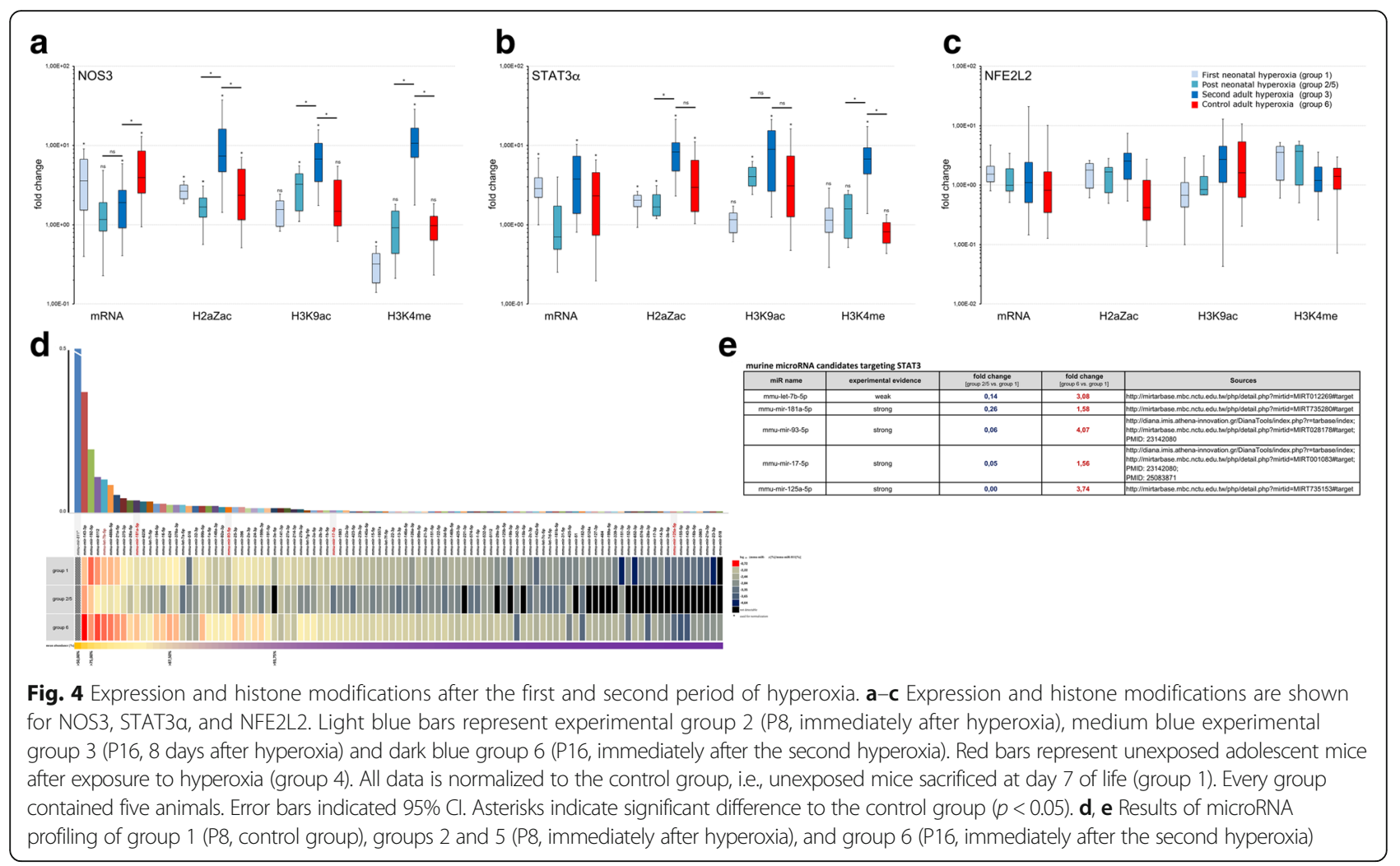

histone acetylation as for example at H3K9, this effect was much weaker in HAEC subjected to repetitive stimulation (Fig. 5). Importantly, we also noticed significant differences between both groups in eNOS and STAT3 $\alpha$ expression. Whereas after repetitive stimulation eNOS (median 0.52, 0.4-0.67) and STAT3 $\alpha$ (median $0.23,0.2-0.29$ ) dropped to levels below baseline measurement levels after 4 weeks (Fig. 6a, c), we noticed a substantial increase in baseline eNOS (median 27.2, 22.335.6) and STAT3 $\alpha$ (median 5.8, 4.8-7.3) expression in HAEC initially subjected to long-term TSA treatment (Fig. 6b, d). Interestingly, the drop to baseline expression of eNOS and STAT3 $\alpha$ in the group with repetitive stimulation took significantly more time compared with the first stimulus.

In the last part of this study, we analyzed the response of the TSA-exposed HAEC to a physiological stimulus. Since in contrast to pulmonary vascular endothelial cells, eNOS expression in peripheral arterial endothelial cells is stimulated by hypoxia, HAEC-either exposed to two 6-h treatments with TSA or one 72-h TSA treatment-were exposed to hypoxia after cultivation for 3 weeks under normal growth conditions. As can be seen in Fig. 7, HAEC with a previous long-term exposure to TSA did show a pathological response to hypoxia with a significant reduction in eNOS expression whereas cells

Table $1 \mathrm{CpG}$ methylation

\begin{tabular}{|c|c|c|c|c|c|c|c|c|c|c|c|c|c|c|c|c|}
\hline & \multicolumn{4}{|c|}{ NANOG } & \multicolumn{6}{|c|}{ NFE2L2 } & \multicolumn{6}{|c|}{ STAT3 } \\
\hline & $\begin{array}{l}\text { CpG } \\
1\end{array}$ & $\begin{array}{l}\text { CpG } \\
2\end{array}$ & $\begin{array}{l}\text { CpG } \\
3\end{array}$ & $\begin{array}{l}\text { CpG } \\
4\end{array}$ & $\begin{array}{l}\text { CpG } \\
1\end{array}$ & $\begin{array}{l}\text { CpG } \\
2\end{array}$ & $\begin{array}{l}\text { CpG } \\
3\end{array}$ & $\begin{array}{l}\text { CpG } \\
4\end{array}$ & $\begin{array}{l}\text { CpG } \\
5\end{array}$ & $\begin{array}{l}\text { CpG } \\
6\end{array}$ & $\begin{array}{l}\text { CpG } \\
1\end{array}$ & $\begin{array}{l}\text { CpG } \\
2\end{array}$ & $\begin{array}{l}\text { CpG } \\
3\end{array}$ & $\begin{array}{l}\text { CpG } \\
4\end{array}$ & $\begin{array}{l}\text { CpG } \\
5\end{array}$ & $\begin{array}{l}\text { CpG } \\
6\end{array}$ \\
\hline $\mathrm{Ctrl}$ & 5.00 & 10.00 & 11.50 & 13.00 & 3.50 & 1.00 & 1.50 & 3.00 & 1.50 & 1.50 & 2.50 & 2.00 & 5.50 & 1.50 & 1.50 & 0.50 \\
\hline Hyperoxia & 5.00 & 11.00 & 12.00 & 12.00 & 3.00 & 1.00 & 1.00 & 2.00 & 1.00 & 1.00 & 3.00 & 1.00 & 3.00 & 1.00 & 1.00 & 1.00 \\
\hline$P[$ Ctrl/hyperoxia $]$ & 0.43 & 0.47 & 0.44 & 0.43 & 0.14 & 0.68 & 0.43 & 0.09 & 0.29 & 0.05 & 0.48 & 0.12 & 0.35 & 0.86 & 0.93 & 0.53 \\
\hline Hyperoxia/5d release & 8.00 & 13.00 & 12.00 & 16.00 & 3.00 & 1.00 & 1.00 & 3.00 & 1.00 & 1.00 & 3.00 & 2.00 & 5.00 & 1.00 & 1.00 & 2.00 \\
\hline$P[C \operatorname{ctr} /$ hyperoxia- & 0.80 & 0.56 & 0.48 & 0.71 & 0.39 & 1.00 & 0.79 & 0.44 & 0.93 & 0.28 & 0.75 & 0.11 & 0.89 & 0.64 & 0.93 & 0.18 \\
\hline
\end{tabular}

release] 


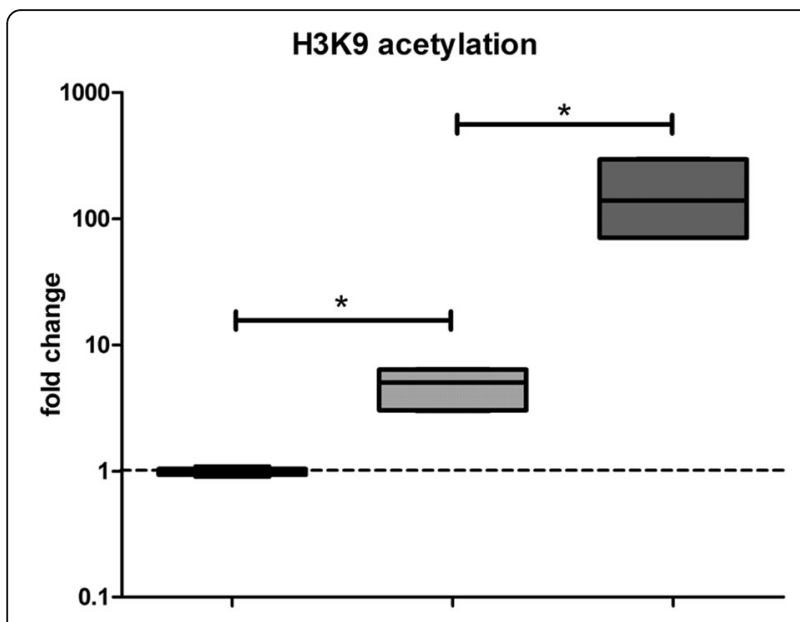

TSA treatment intervals

Fig. $5 \mathrm{H} 3 \mathrm{~K} 9$ acetylation at the NOS3 gene locus after TSA treatment. HAEC were exposed to either trichostatin A (TSA) twice for $6 \mathrm{~h}$ with and interval of $60 \mathrm{~h}$ (light gray) or once for $72 \mathrm{~h}$. Histone modification was analyzed using CHiP immediately after the TSA exposure and compared to unexposed HAEC (black). All experiments were performed in triplicates. Error bars indicated $95 \% \mathrm{Cl}$. Asterisks indicate significant differences $(p<0.05)$

with a previous short-term repetitive exposure to TSA showed a response pattern very similar to control HAEC. This pathological response was accompanied by altered histone modifications at the NOS3 gene locus (Fig. 7d, e). Importantly, as previously mentioned, HAEC with previous long-term exposure to TSA showed persistence of H3K9ac at the NOS3 gene locus whereas in cells with short-term repetitive TSA treatment histone marks at the NOS3 gene locus were restored. Interestingly, we also noticed high levels of the repressive histone mark Н3К27me3 which persisted during hypoxia.

\section{Discussion}

Bronchopulmonary dysplasia (BPD) remains one of the main problems of prematurity even in the postsurfactant area. Importantly, BPD is also the main reason for pulmonary hypertension in the pediatric population. Early oxygen exposure has been identified as one of the most important risk factors for the development of BPD. Even though the exact mechanism remains unclear, it seems to involve high oxygen exposure possibly by inducing excessive pulmonary vasodilatation and production of oxygen radicals followed by tissue damage. However, to date, no pathophysiological link has been identified between early oxygen exposure and later pathological response patterns of the pulmonary vasculature in pulmonary hypertension.

Here, we provide the first evidence that excessive oxygen exposure early in life leads to permanent changes in the epigenetic signatures at the NOS3 locus in pulmonary endothelial artery cells in a mouse model. These changes seem to be associated with a non-physiological expression pattern of eNOS upon oxygen exposure later in life. Moreover, non-physiological alterations of epigenetic signatures at the NOS3 gene locus induce similar effects in human aortic endothelial cells (HAEC) in vitro.

Specifically, in the first part of the study, we demonstrated that NOS3 mRNA levels increased in pulmonary artery cells upon neonatal oxygen exposure in newborn mouse. This was paralleled by hyperemia and later disruption of the alveolar structure as shown by histology. This is in line with previous reports demonstrating increased protein concentrations [7] and increased NOS3 mRNA levels upon early excessive oxygen exposure in various models [26] and human tissue samples [27]. Interestingly, we did not observe any changes in RNA expression of NFE2L2, a transcription factor that regulates the expression of antioxidant proteins that protect against oxidative damage triggered by injury and inflammation. This fits to the previous observation that SOD3, an Nrf2-independent antioxidant, was not found to be upregulated as one might expect in the $\mathrm{O}_{2}$-exposed neonatal mice compared with room air [28]. In fact, this possibly reflects the inefficiency of counteractive antioxidant mechanisms in the neonatal lung.

The observed increase of NOS3 mRNA levels was paralleled by an increase in $\mathrm{H} 3 \mathrm{~K} 9$ and $\mathrm{H} 2 \mathrm{Az}$ acetylation very similar to our previous observations in HUAEC [18]. Importantly, we did not observe a restitution of acetylation patterns at the NOS 3 gene locus after 5 days of normoxia. Moreover, upon a second exposure to hyperoxia later in life, we noticed a dramatic increase in $\mathrm{H} 3 \mathrm{~K} 9$ and $\mathrm{H} 2 \mathrm{Az}$ acetylation and also H3K4 methylation in mice that have been exposed to neonatal hyperoxia. However, NOS3 mRNA levels did not increase but were even found to be substantial lower in these mice when compared to healthy controls. Obviously, the substantial increase in activating histone marks at the NOS3 gene locus did not lead to an increase in transcription in these mice. This is in line with a previous report demonstrating a suppressive effect on NOS3 mRNA synthesis after $12 \mathrm{~h}$ incubation with TSA [29]. As we have previously shown the most reasonable explanation for this phenomenon is an increased activity of counteracting processes mainly due to a negative feedback loop, which in humans presumably involves a co-processed 27 nt-ncRNA encoded in NOS3 intron 5 $[16,30]$, which targets and suppresses STAT3 mRNA [31]. Our analyses allow us to speculate that a negative regulatory function in mice, where a STAT3-targeting 27 nt-RNA was not identified, could be fulfilled by one or several of the differentially expressed miRs, which we identified by microRNA expression profiling. 


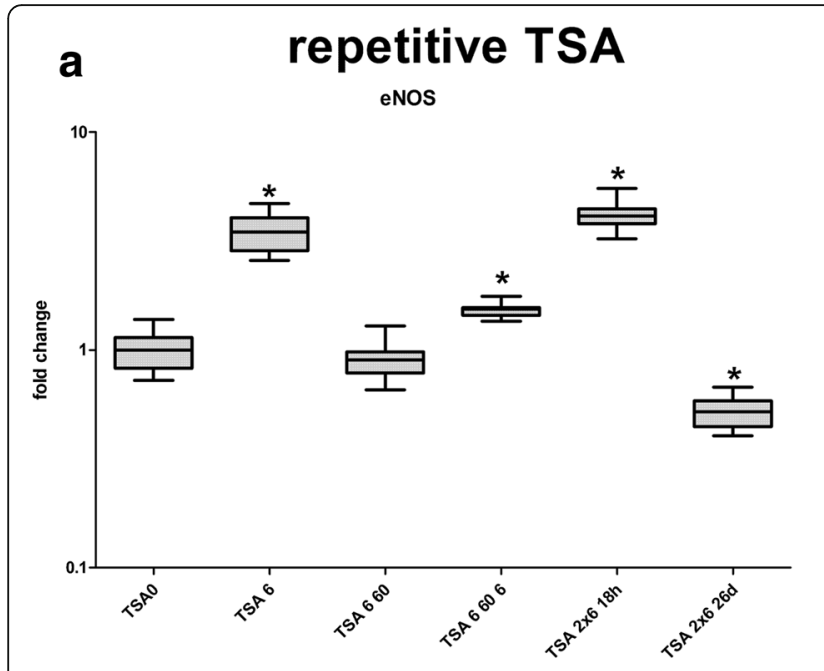

b

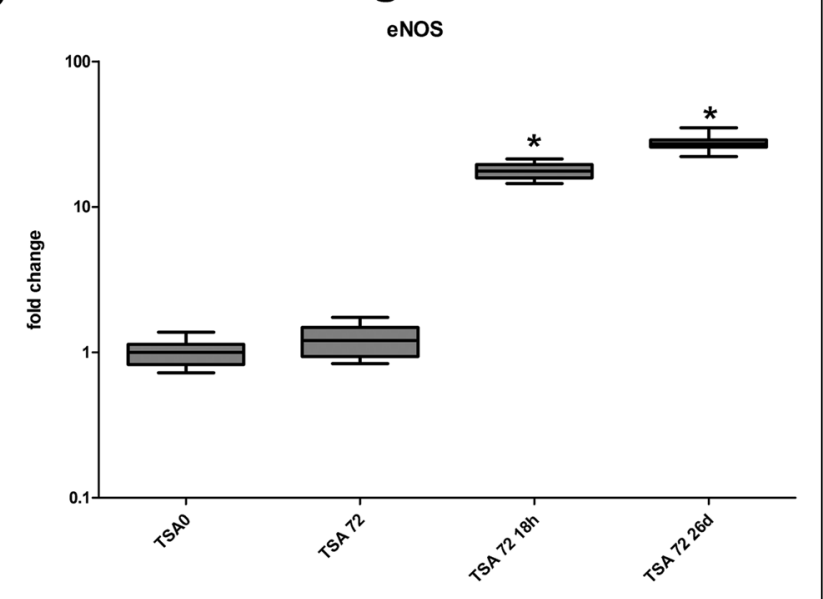

C

STAT3 $\alpha$

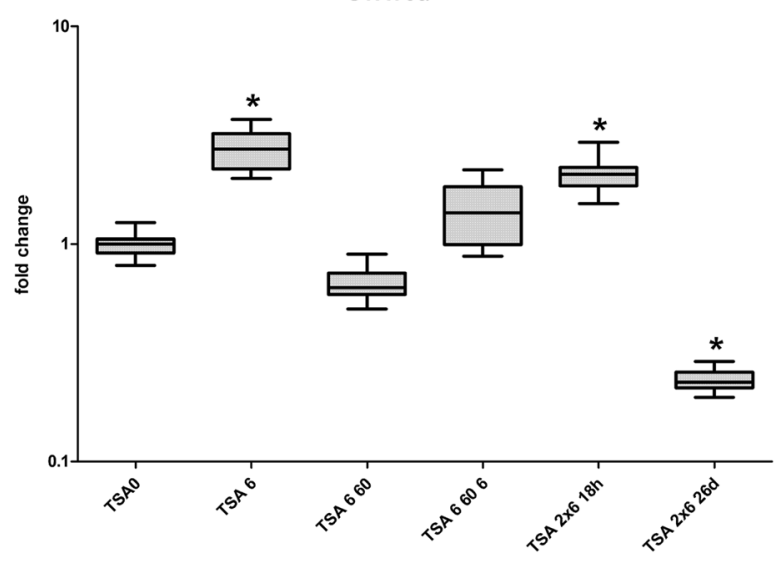

d

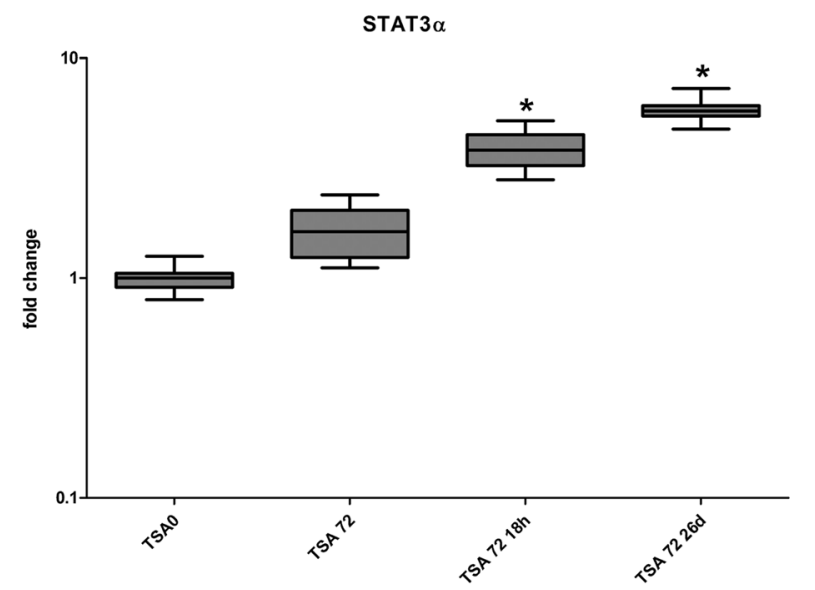

Fig. $\mathbf{6}$ eNOS and STAT3a expression in HAEC after TSA treatment. a, c HUAEC were treated twice with TSA for $6 \mathrm{~h}$ with an interval in between of 60 h. b. d HAEC were treated once with TSA for 72 h. eNOS and STAT3a expression was analyzed at different time points. All experiments were performed three times with three different HAEC lines. Error bars indicated 95\% Cl. Asterisks indicate significant differences $(p<0.05)$

Moreover, we also observed increased levels of H3K27me3-a repressive histone mark-which might function as an additional counteracting process responsible for the absent increase in NOS3 mRNA levels. Considering a recent report demonstrating eNOS uncoupling and decreased NO production with an enhanced superoxide production in adult rats after neonatal hyperoxia [32], the observed downregulation of NOS3 mRNA production in our study might be a protective mechanism downregulating eNOS expression upon oxygen exposure to prevent further superoxide production and tissue damage. Overall, the observed changes in epigenetic signatures are in line with recent reports such as the study by Zhu et al. who found that H3K27 trimethylation is present in a BPD mouse model and involved in RUNX3 downregulation, a gene associated with pulmonary development [33].
In the second part of the study, we provide further evidence for the relevance of the observed histone modifications for the regulation of NOS3 expression using an in vitro cell culture model. For correct interpretation of the data, it is important to mention that the physiological response pattern of human peripheral arterial endothelial cells is opposite to the response pattern of pulmonary endothelial artery cells, i.e., vasodilatation and NOS3 upregulation upon hypoxia and not hyperoxia. To induce a response pattern comparable to the mouse model upon hyperoxia, we therefore subjected HAEC to hypoxia after exposure to TSA. Similar to the mouse model, we observed long persistence of altered acetylation patterns after long-term exposure to TSA whereas histone signatures were restored after repetitive short-term exposure. In HAEC with long-term exposure to TSA, NOS3 mRNA levels remained high corresponding to an increase $\mathrm{H} 3 \mathrm{~K} 9 \mathrm{ac}$ at the NOS3 gene locus. 


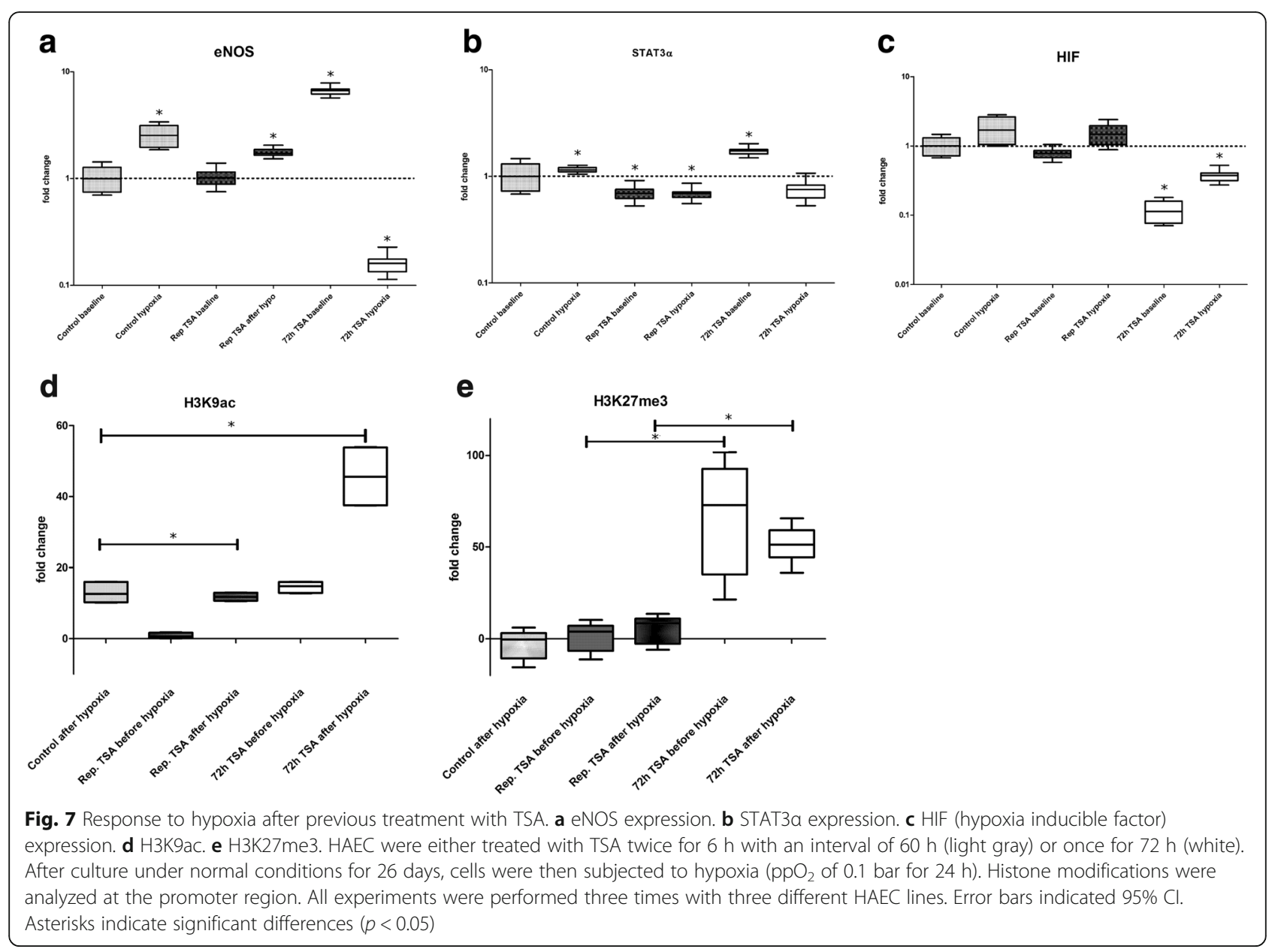

However, upon exposure to hypoxia, we observed a substantial decrease in NOS3 mRNA levels in these cells even though levels or H3K9ac further increased. Currently, similar to the constant increase in H3K27me3 at the NOS3 gene locus in these cells, we have no good explanation for this phenomenon. Possibly, this just reflects a complete disturbance and malfunction of the epigenetic code, but this needs to be further elucidated.

One major limitation of our study is the lack of functional studies such as measurement of NO. Since many previous studies have already demonstrated that upregulation of NOS3 corresponds very well with increased NO production in the hyperoxia mouse model [7], we believe that mRNA levels are sufficient physiological to characterize the cellular response patterns. In addition, even if that is not the case, it does not change the disturbances observed on the level of mRNA expression and in epigenetic signatures. The second major limitation of this study is the lack of further functional analysis on the role of miRNA on the development of BPD. However, since this seems to be rather complex [34] and considering the focused rather than generalistic approach of this study, this would have been beyond the primary scope of this study.

\section{Conclusions}

This study provides further evidence for the longterm relevance of early life events on changes in epigenetic signatures using a newborn mouse model of hyperoxia-induced BPD. It demonstrates the occurrence and relevance of persisting epigenetic marks for regulation of vascular endothelial response patterns upon physiological stimuli later in life. Even though currently very theoretical, this suggests that treatment of BPD and its sequelae by modification of epigenetic mechanisms might at least be an option.

\section{Abbreviations}

BPD: Bronchopulmonary dysplasia; BW: Birth weight; eNOS: Endothelial nitric oxide synthase; GA: Gestational age; HAEC: Human arterial endothelial cell; HDAC: Histone deacetylase; HOX: Hyperoxia; NO: Nitric oxide;

NOX: Normoxia; PCR: Polymerase chain reaction; TSA: Trichostatin A

\section{Acknowledgements}

We would like to sincerely thank Claudia Förster and Prof. Klaus-Peter Zimmer for their constant support. 


\section{Funding}

Rhea van den Bruck received an internal research grant from the University of Witten/Herdecke.

\section{Availability of data and materials}

Not applicable.

\section{Authors' contributions}

AJ, CC, SM, JM, LK, PW, MA, and RB have performed data acquisition and analyzed the data. AJ and CC wrote the manuscript. AJ, CC, and JP took part in the main study design and supervision of the study. SB took part in the supervision of the study. SB and JP critically reviewed the manuscript. All authors read and approved the final manuscript.

\section{Ethics approval}

All animal experiments were performed in accordance with the National Institutes of Health Guidelines for the Use of Laboratory Animals. Animal experiments were approved by the Federal Authorities for Animal Research of the Regierungspräsidium Giessen, Hessen, Germany, protocol 105/2011. This study was conducted with the approval of the Witten/Herdecke University Ethics board.

\section{Competing interests}

All authors declare that they have no competing interests.

\section{Publisher's Note}

Springer Nature remains neutral with regard to jurisdictional claims in published maps and institutional affiliations.

\begin{abstract}
Author details
${ }^{1}$ Excellence Cluster Cardio-Pulmonary System (ECCPS), Member of the German Center for Lung Research (DZL), Department of Internal Medicine II, Universities of Giessen and Marburg Lung Center (UGMLC), Aulweg 130, 35392 Giessen, Germany. ${ }^{2}$ University Children's Hospital Gießen, Division of General Pediatrics and Neonatology, Justus-Liebig-University, Gießen, Germany. ${ }^{3}$ Department of Pediatrics, HELIOS Medical Center Wuppertal, Center for Clinical \& Translational Research (CCTR), Center for Biomedical Education \& Research (ZBAF), Witten/Herdecke University, Wuppertal, Germany. ${ }^{4}$ EKO Children's Hospital, Oberhausen, Witten/Herdecke University, Alfred-Herrhausen Str. 40, Witten, Germany.
\end{abstract}

Received: 31 July 2017 Accepted: 9 March 2018

Published online: 20 March 2018

\section{References}

1. Northway WH Jr, Rosan RC, Porter DY. Pulmonary disease following respirator therapy of hyaline-membrane disease. Bronchopulmonary dysplasia. N Engl J Med. 1967;276(7):357-68.

2. Stoll BJ, Hansen NI, Bell EF, Shankaran S, Laptook AR, Walsh MC, Hale EC, Newman NS, Schibler K, Carlo WA, et al. Neonatal outcomes of extremely preterm infants from the NICHD Neonatal Research Network. Pediatrics. 2010;126(3):443-56.

3. Baraldi E, Filippone M. Chronic lung disease after premature birth. N Engl J Med. 2007:357(19):1946-55

4. Bhandari A, Bhandari V. Pitfalls, problems, and progress in bronchopulmonary dysplasia. Pediatrics. 2009;123(6):1562-73.

5. Shaw GM, O'Brodovich HM. Progress in understanding the genetics of bronchopulmonary dysplasia. Semin Perinatol. 2013:37(2):85-93.

6. Vento M, Moro M, Escrig R, Arruza L, Villar G, Izquierdo I, Roberts LJ 2nd, Arduini A, Escobar JJ, Sastre J, et al. Preterm resuscitation with low oxygen causes less oxidative stress, inflammation, and chronic lung disease. Pediatrics. 2009;124(3):e439-49.

7. Potter CF, Kuo NT, Farver CF, McMahon JT, Chang CH, Agani FH, Haxhiu MA, Martin RJ. Effects of hyperoxia on nitric oxide synthase expression, nitric oxide activity, and lung injury in rat pups. Pediatr Res. 1999;45(1):8-13.

8. Kondrikov D, Elms S, Fulton D, Su Y. eNOS-beta-actin interaction contributes to increased peroxynitrite formation during hyperoxia in pulmonary artery endothelial cells and mouse lungs. J Biol Chem. 2010;285(46):35479-87.

9. Simpson SJ, Hall GL, Wilson AC. Lung function following very preterm birth in the era of 'new' bronchopulmonary dysplasia. Respirology. 2015;20(4):535-40.
10. Mourani PM, Sontag MK, Younoszai A, Miller Jl, Kinsella JP, Baker CD Poindexter BB, Ingram DA, Abman SH. Early pulmonary vascular disease in preterm infants at risk for bronchopulmonary dysplasia. Am J Respir Crit Care Med. 2015;191(1):87-95.

11. O'Reilly MA, Yee M, Buczynski BW, Vitiello PF, Keng PC, Welle SL, Finkelstein JN, Dean DA, Lawrence BP. Neonatal oxygen increases sensitivity to influenza A virus infection in adult mice by suppressing epithelial expression of Ear1. Am J Pathol. 2012;181(2):441-51.

12. Domm W, Misra RS, O'Reilly MA. Affect of early life oxygen exposure on proper lung development and response to respiratory viral infections. Front Med. 2015;2:55.

13. Bhandari V, Bizzarro MJ, Shetty A, Zhong X, Page GP, Zhang H, Ment LR, Gruen JR, Neonatal Genetics Study G. Familial and genetic susceptibility to major neonatal morbidities in preterm twins. Pediatrics. 2006;117(6):1901-6.

14. Londhe VA, Sundar IK, Lopez B, Maisonet TM, Yu Y, Aghai ZH, Rahman I. Hyperoxia impairs alveolar formation and induces senescence through decreased histone deacetylase activity and up-regulation of p21 in neonatal mouse lung. Pediatr Res. 2011:69(5 Pt 1):371-7.

15. Ni W, Lin N, He H, Zhu J, Zhang Y. Lipopolysaccharide induces upregulation of TGF-alpha through HDAC2 in a rat model of bronchopulmonary dysplasia. PLoS One. 2014;9(3):e91083.

16. Postberg J, Kanders M, Forcob S, Willems R, Orth V, Hensel KO, Weil PP, Wirth $\mathrm{S}$, Jenke AC. CpG signalling, H2A.Z/H3 acetylation and microRNAmediated deferred self-attenuation orchestrate foetal NOS3 expression. Clin Epigenetics. 2015;7(1):9.

17. Jenke AC, Postberg J, Raine T, Nayak KM, Molitor M, Wirth S, Kaser A, Parkes $M$, Heuschkel RB, Orth $V$, et al. DNA methylation analysis in the intestina epithelium-effect of cell separation on gene expression and methylation profile. PLoS One. 2013:8(2):e55636.

18. Postberg J, Kanders M, Forcob S, Willems R, Orth V, Hensel KO, Weil PP, Wirth S, Jenke AC. CpG signalling, H2A.Z/H3 acetylation and microRNAmediated deferred self-attenuation orchestrate foetal NOS3 expression. Clin Epigenetics. 2015;7:9.

19. Weil PP, Jaszczyszyn Y, Baroin-Tourancheau A, Postberg J, Amar L. Holistic and affordable analyses of MicroRNA expression profiles using tagged cDNA libraries and a multiplex sequencing strategy. Methods Mol Biol. 2017;1654:179-96.

20. Blankenberg D, Von Kuster G, Coraor N, Ananda G, Lazarus R, Mangan M, Nekrutenko A, Taylor J. Galaxy: a web-based genome analysis tool for experimentalists. Curr Protoc Mol Biol. 2010;Chapter 19(Unit 19 10):11-21.

21. Giardine B, Riemer C, Hardison RC, Burhans R, Elnitski L, Shah P, Zhang Y, Blankenberg D, Albert I, Taylor J, et al. Galaxy: a platform for interactive large-scale genome analysis. Genome Res. 2005;15(10):1451-5.

22. Goecks J, Nekrutenko A, Taylor J, Galaxy T. Galaxy: a comprehensive approach for supporting accessible, reproducible, and transparent computational research in the life sciences. Genome Biol. 2010;11(8):R86.

23. Vitsios DM, Enright AJ. Chimira: analysis of small RNA sequencing data and microRNA modifications. Bioinformatics. 2015;31(20):3365-7.

24. Thebaud B. Angiogenesis in lung development, injury and repair: implications for chronic lung disease of prematurity. Neonatology. 2007;91(4):291-7.

25. Thebaud B, Abman SH. Bronchopulmonary dysplasia: where have all the vessels gone? Roles of angiogenic growth factors in chronic lung disease. Am J Respir Crit Care Med. 2007:175(10):978-85.

26. Feng X, Guo Z, Nourbakhsh M, Hauser H, Ganster R, Shao L, Geller DA. Identification of a negative response element in the human inducible nitric-oxide synthase (hiNOS) promoter: the role of NF-kappa B-repressing factor (NRF) in basal repression of the hiNOS gene. Proc Natl Acad Sci U S A. 2002:99(22):14212-7.

27. Davis CW, Gonzales LW, Ballard RA, Ballard PL, Guo C, Gow AJ. Expression of nitric oxide synthases and endogenous $\mathrm{NO}$ metabolism in bronchopulmonary dysplasia. Pediatr Pulmonol. 2008;43(7):703-9.

28. Poonyagariyagorn HK, Metzger S, Dikeman D, Mercado AL, Malinina A, Calvi C, McGrath-Morrow S, Neptune ER. Superoxide dismutase 3 dysregulation in a murine model of neonatal lung injury. Am J Respir Cell Mol Biol. 2014;51(3):380-90.

29. Rossig L, Li H, Fisslthaler B, Urbich C, Fleming I, Forstermann U, Zeiher AM Dimmeler $\mathrm{S}$. Inhibitors of histone deacetylation downregulate the expression of endothelial nitric oxide synthase and compromise endothelial cell function in vasorelaxation and angiogenesis. Circ Res. 2002;91(9):837-44

30. Zhang MX, Ou H, Shen YH, Wang J, Wang J, Coselli J, Wang XL. Regulation of endothelial nitric oxide synthase by small RNA. Proc Natl Acad Sci U S A. 2005:102(47):16967-72

31. Yan L, Hao H, Elton TS, Liu Z, Ou H. Intronic microRNA suppresses endothelial nitric oxide synthase expression and endothelial cell proliferation via inhibition of STAT3 signaling. Mol Cell Biochem. 2011;357(1-2):9-19. 
32. Yzydorczyk C, Comte B, Huyard F, Cloutier A, Germain N, Bertagnolli M, Nuyt AM. Developmental programming of eNOS uncoupling and enhanced vascular oxidative stress in adult rats after transient neonatal oxygen exposure. J Cardiovasc Pharmacol. 2013;61(1):8-16.

33. Zhu Y, Fu J, Yang H, Pan Y, Yao L, Xue X. Hyperoxia-induced methylation decreases RUNX3 in a newborn rat model of bronchopulmonary dysplasia. Respir Res. 2015;16:75.

34. Nardiello C, Morty RE. MicroRNA in late lung development and bronchopulmonary dysplasia: the need to demonstrate causality. Mol Cell pediatr. 2016;3(1):19.

Submit your next manuscript to BioMed Central and we will help you at every step:

- We accept pre-submission inquiries

- Our selector tool helps you to find the most relevant journal

- We provide round the clock customer support

- Convenient online submission

- Thorough peer review

- Inclusion in PubMed and all major indexing services

- Maximum visibility for your research

Submit your manuscript at www.biomedcentral.com/submit
Biomed Central 